\title{
H5N1 Virus Hemagglutinin Inhibition of CAMP-Dependent CFTR via TLR4-Mediated Janus Tyrosine Kinase 3 Activation Exacerbates Lung Inflammation
}

\author{
Ke Cao," Minhui Chen," Xiang Jie, Yansheng Wang, Qiasheng Li, and Jun Xu
}

State Key Laboratory of Respiratory Diseases, Guangzhou Institute of Respiratory Diseases, The First Affiliated Hospital of Guangzhou Medical University, Guangzhou Medical University, Guangzhou, People's Republic of China

\begin{abstract}
The host tolerance mechanisms to avian influenza virus (H5N1) infection that limit tissue injury remain unknown. Emerging evidence indicates that cystic fibrosis transmembrane conductance regulator (CFTR), a cAMP-dependent $\mathrm{Cl}^{-} \mathrm{channel}$, modulates airway inflammation. Janus tyrosine kinase (JAK) 3, a JAK family member that plays a central role in inflammatory responses, prominently contributes to the dysregulated innate immune response upon H5N1 attachment; therefore, this study aims to elucidate whether JAK3 activation induced by H5N1 hemagglutinin (HA) inhibits CAMP-dependent CFTR channels. We performed short-circuit current, immunohistochemistry and molecular analyses of the airway epithelium in Jak $3^{+/+}$and Jak $3^{+/-}$mice. We demonstrate that H5NI HA attachment inhibits cAMP-dependent CFTR $\mathrm{Cl}^{-}$channels via JAK3-mediated adenylyl cyclase (AC) suppression, which reduces CAMP production. This inhibition leads to increased nuclear factor-kappa B (NF-kB) signaling and inflammatory responses. H5N1 HA is detected by TLR4 expressed on respiratory epithelial cells, facilitating JAK3 activation. This activation induces the interaction between TLR4 and Gai protein, which blocks ACs. Our findings provide novel insight into the pathogenesis of acute lung injury via the inhibition of CAMP-dependent CFTR channels, indicating that the administration of cAMP-elevating agents and targeting JAK3 may activate host tolerance to infection for the management of influenza virus-induced fatal pneumonia.
\end{abstract}

Online address: http://www.molmed.org

doi: $10.2119 / \mathrm{molmed} .2014 .00189$

\section{INTRODUCTION}

Increasing evidence indicates that superinflammation, a consequence of an exacerbated innate immune response, plays a critical role $(1,2)$ in the rapid progression to adult respiratory distress syndrome (ARDS) following H5N1 infection; however, innate immune mechanisms protect the infected host by reducing the viral burden. Thus, an optimal immune response is characterized by a balance between efficient pathogen clearance and an acceptable level of im- munopathology $(3,4)$. Tolerance to infection has recently been found to constitute a distinct strategy of host defense that limits tissue damage, facilitating a higher magnitude and duration of the immune response (5). The elucidation of the inducible tolerance mechanism in response to $\mathrm{H} 5 \mathrm{~N} 1$ infection will provide new therapeutic strategies for severe lung damage mediated by viral attack by attaining an optimal immune response.

The mechanisms that typically maintain the homeostasis of various physio-

"KC and MC contributed equally to this work.

Address correspondence to Jun Xu, Guangzhou Institute of Respiratory Diseases, 151 Yanjiang Road, Guangzhou 510120, China. Phone: +86-(20)-34298187; Fax: +86-(20)-83062729; E-mail: xufeili@vip. 163.com.

Submitted September 22, 2014; Accepted for publication January 12, 2015; Published Online (www.molmed.org) January 12, 2015.

The Feinstein Institute for Medical Research

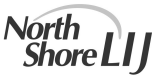

Empowering Imagination. Pioneering Discovery.

logical systems are likely to contribute to host tolerance to infection $(6,7)$. Cystic fibrosis transmembrane conductance regulator (CFTR) is a cyclic adenosine monophosphate (cAMP)-dependent $\mathrm{Cl}^{-}$ channel expressed at the apical membrane of epithelial cells lining the tracheobronchial tree. CFTR channels secrete $\mathrm{Cl}^{-}$and mucus simultaneously with $\mathrm{Ca}^{2+}$ activated $\mathrm{Cl}^{-}$channels (CaCCs) (8) and regulate the amiloride-sensitive epithelial $\mathrm{Na}^{+}$channel $(\mathrm{ENaC})$, constituting the pathways that restrict $\mathrm{Na}^{+}$and mucus absorption (9). Coordinating the regulation of the secretion and absorption of $\mathrm{NaCl}$ and fluids and airway surface liquid (ASL) homeostasis is crucial. Changing the salt and water composition of the periciliary fluid causes isotonic dehydration of the airway surface, leading to an impairment of mucociliary clearance. In the case of the $\triangle$ F508-CFTR mutation, constitutive nuclear factor-kappa B (NF-кB) activation results in IL-8-mediated chronic neutrophilic lung disease $(10,11)$. Several 
investigators in the field believe that airway inflammation in CF is secondary to persistent bacterial infection, which results from impaired mucociliary clearance; however, recent evidence supports the hypothesis that the dysregulation of the inflammatory response is an intrinsic component of the CF phenotype and that airway inflammation may occur prior to or in the absence of bacterial infection. Moreover, numerous in vitro studies using human epithelial CF and control cell lines have confirmed that CFTR is an important inflammatory regulator and that CFTR mutations are associated with both the constitutive activation of proinflammatory signaling, especially NF-кB, in the absence of any apparent microbial stimulus and the exaggerated responses to bacterial products $(12,13)$.

We previously demonstrated that exposure to hemagglutinin (HA), the surface glycoprotein of $\mathrm{H} 5 \mathrm{~N} 1$ that is indispensable for viral receptor binding, fusion, transmission, virulence and pathology (14-16), induced the activation of IFNindependent JAK/STAT and NF-кB in pulmonary epithelial cells. This activation was accompanied by the elevated secretion of chemokines/cytokines, including IP-10, IL-6, IL-8, MCP-1, MIP-1 $\alpha$, MIP-1 $\beta$ and RENTES. Furthermore, Janus tyrosine kinase (JAK) 3, a member of the JAK family of tyrosine kinases involved in cytokine receptor-mediated intracellular signal transduction, is a molecular determinant in the H5N1 HA-induced dysregulated innate immune response. The inhibition of JAK3 activation causes the negative regulation of NF- $\mathrm{\kappa B}$ signaling (17); therefore, we speculated that $\mathrm{H} 5 \mathrm{~N} 1$ HA-stimulated NF-кB signaling may be, at least in part, attributed to the disturbance of epithelial CFTR inflammatory regulation, a host tolerance mechanism to infection, via a JAK3 activationdependent response. The present study reveals for the first time that H5N1 HA attachment to the airway epithelia stimulates the JAK3 signaling cascade, leading to the suppression of CFTR inflammatory regulation and exaggerating innate immune responses.

\section{MATERIALS AND METHODS}

\section{Preparation of the HA Protein}

The recombinant HA protein from H5N1 (A/chicken/Guangdong/191/04, GenBank: AY737289) was generated as described previously (17).

\section{Animal Models}

B6129S4-Jak3 $^{\text {tm1Lj }}\left(\mathrm{JAK}^{-/-}\right)$mice and wild-type B6129SF2/J $\left(\mathrm{JAK}^{+/+}\right.$) mice (6 to $8 w k s$ of age) are all from the C57BL/ 6 mouse genetic background and were purchased from the Jackson Laboratory (Bar Harbor, ME, USA). JAK3 heterozygous knockout $\left(\mathrm{JAK}^{+/-}\right)$mice were generated by crossing $\mathrm{JAK}^{-/-}$mice with $\mathrm{JAK}^{+/+}$mice. Then, the genotypes were identified via PCR. All mice were housed at a constant temperature $\left(20^{\circ} \mathrm{C}\right)$ with a 12-h light-dark photoperiod and allowed food and water ad libitum. All procedures were performed in compliance with the National Institutes of Health-adopted Guide for Care and Use of Laboratory Animals (18) and were approved by the Bioethics Committee of State Key Laboratory of Respiratory Disease, Guangzhou Medical University. JAK3 ${ }^{+/+}$mice were intratracheally administered saline; HA (1 mg/kg) with or without pretreatment with either forskolin (10 mg/kg, SigmaAldrich, St. Louis, MO, USA) or JAK3 inhibitor VI (0.15 mg/kg, Calbiochem, Darmstadt, Germany) by intraperitoneal (IP) injection; or HA $(0.5 \mathrm{mg} / \mathrm{kg})$ with or without pretreatment with glibenclamide IP injection (10 mg/kg, Sigma-Aldrich). $\mathrm{JAK}^{+/-}$mice were administered HA at a dose of $1 \mathrm{mg} / \mathrm{kg}$. Pulmonary histopathology and immunostaining were performed $12 \mathrm{~h}$ after the preceding treatment.

\section{Cell Culture and Treatment}

Cultured 16HBE and calu-3 cells were treated with saline, JAK3 inhibitor VI (760 nmol/L), TLR4 inhibitor (candesartan, $5 \mu \mathrm{mol} / \mathrm{L}, 3 \mathrm{~B}$ Scientific Corporation, Wuhan, China), forskolin (10 $\mu \mathrm{mol} / \mathrm{L})$ or glibenclamide $(500 \mu \mathrm{mol} / \mathrm{L})$ for $30 \mathrm{~min}$ prior to HA stimulation and then subjected to different experiments. To test
Gai-mediated inhibition of AC, 16HBE cells were pretreated with IBMX (1 mmol/L, Sigma-Aldrich) and forskolin and either HA alone or HA in combination with IL-2 (100 UI/mL). In some experiments, cells were pretreated with pertussis toxin (100 ng/mL, SigmaAldrich) for $16 \mathrm{~h}$ or a TLR4 inhibitor or JAK3 inhibitor VI for $30 \mathrm{~min}$.

\section{CFTR-Dependent Short-Circuit Current Measurements}

Tracheas of $\mathrm{JAK}^{+/-}$or $\mathrm{JAK}^{+/+}$mice that had or had not received JAK3 inhibitor VI and TLR4 inhibitor (100 mg/kg) were pretreated with exposure of apical membranes to H5N1 HA, and then mounted in an Ussing chamber bathed in Krebs-Henseleit (K-H) solution to perform short-circuit current assay by using a VCC MC6 voltage-current clamp amplifier (VCC MC6, Physiologic Instrument, San Diego, CA, USA). The data were displayed on a signal collection and analysis system (Acquire \& Analyze Rev II, San Diego, CA, USA) (19). Forskolin $(10 \mu \mathrm{mol} / \mathrm{L})$ was used to induce anion secretion via an increase in cAMP levels. $\mathrm{Cl}^{-}$free $\mathrm{KH}$ solution was employed to examine the effects on $\mathrm{Cl}^{-}$current. To inhibit electrically conductive $\mathrm{Na}^{+}$transport, amiloride $(100 \mu \mathrm{mol} / \mathrm{L})$ was added in all studies.

\section{Intracellular CAMP and Adenylyl Cyclase (AC) Assays}

cAMP and AC levels were measured in cells using a cAMP assay kit (Assay Designs Inc., Ann Arbor, MI, USA) and an AC assay kit (Uscn Life Science Inc., Wuhan, China), respectively. The levels were corrected to the total protein levels, and the data were expressed as picomoles per milligram of protein.

\section{Immunofluorescent Staining}

Frozen lung tissue sections and cells cultured on glass coverslips were subjected to immunofluorescent staining for evaluation of CFTR expression or NF-кB/ JAK3 activation using primary antibodies against CFTR (Abcam, Cambridge, MA, USA), phosphorylated NF-кBp65 (Cell 
Signaling Technology Inc., Beverly, MA, USA) and phosphorylated JAK3 (Santa Cruz Biotechnology, Santa Cruz, CA, USA), respectively. The slides were then incubated with species-matched fluorescent secondary antibodies and DAPI (for nuclear staining). Images were captured using a Nikon C1 Si confocal system (Nikon Corporation, Tokyo, Japan).

\section{Lung Histology}

Lung histology was performed as previously described (17).

\section{Luminex Assay}

Cytokine and chemokine production levels in supernatants were analyzed using a Luminex assay LiquidChip system (Panomics, Santa Clara, CA, USA) as previously described (17).

\section{Western Blot}

Western blotting for examination of JAK3 activity or CFTR expression was performed as previously described (17). The intensities of the relevant bands were quantified using ImageJ software (National Institutes of Health, Bethesda, MD, USA).

\section{Quantitative Reverse-Transcription PCR (RT-PCR) Analysis}

Cellular RNA was extracted and reverse transcribed to cDNA using the M-MLV first strand kit (Invitrogen, Life Technologies [Thermo Fisher Scientific Inc., Waltham, MA, USA]). Quantitative real-time PCR was performed to evaluate CFTR or TLR4 gene expression using SYBR Premix Ex Taq II (TaKaRa, Dalian, China). Fold changes of gene expression were normalized to $\beta$-actin and were quantified by the change-in-threshold method $(\Delta \Delta C \mathrm{t})$. Primer sequences for the genes of interest were designed using TAKARA Biotechnology. The primer sequences are listed below:

CFTR forward primer, 5'-TTAAAGCTGT CAAGCCGTGTTC-3';

CFTR reverse primer, 5'-GCCAATGCAA GTCCTTCATCA-3';

TLR4 forward primer, 5'-AGAAC CTGGACCTGAGCTTTAATC-3';
TLR4 reverse primer, 5'-GAGGTGGCTT AGGCTCTGATATG-3';

$\beta$-actin forward primer, 5'-CCTGG

CACCCAGCACAAT-3';

$\beta$-actin reverse primer, 5'-GCTGATCCAC ATCTGCTGGAA-3'.

\section{Statistical Analysis}

All of the experimental data shown are expressed as the means \pm S.D. and were repeated at least three times, unless otherwise indicated. Statistical analysis was performed using one-way analysis of variance (ANOVA) followed by Student $t$ test, and $p<0.05$ was considered to be significant.

All supplementary materials are available online at www.molmed.org.

\section{RESULTS}

H5N1 HA-Induced Activation of JAK3 Inhibits CAMP-Dependent CFTR in the Airway Epithelium by Blocking AC

As predicted, treatment with HA (40 ug/mL) for 10 or 20 min resulted in a remarkable decline in the basal shortcircuit current (Isc) of the apical membrane of the airway epithelium in the wild-type $\left(\mathrm{JAK}^{+/+}\right.$) mice but did not induce this change in the $\mathrm{JAK}^{+/-}$mice. The addition of a single dose of the cAMP agonist forskolin to the microUssing perfusion chamber caused an increase in the basal Isc ( $\Delta \mathrm{Isc})$ of the murine tracheal tissues, as expected. However, with HA pretreatment, we detected an additional increase in Isc ( $\Delta \mathrm{Isc})$ in the $\mathrm{JAK}^{+/+}$mice compared with $\mathrm{JAK}^{+/-}$mice, in response to forskolin (Figure 1A), but did not find the change under the condition of $\mathrm{Cl}^{-}$free-KH perfusion (Supplementary Figure S1A). Exposure of tracheal tissues of $\mathrm{JAK}^{+/+}$ mice that had received JAK3 inhibitor VI to HA reversed this HA-induced Isc decline, and $\Delta \mathrm{Isc}$ returned to basal levels in response to forskolin (Figure 1B). We observed that H5N1 HA had no significant effect on amiloride-sensitive sodium current after exposure of the tracheal epithelium to HA for the indicated time (Supplementary Figure S1B).
The ELISA results indicate that HA stimulation in 16HBE cells inhibited AC activity, resulting in a significant reduction in intracellular cAMP levels. This inhibition was attenuated in cells pretreated with JAK3 inhibitor VI (Figures $2 \mathrm{~A}, \mathrm{~B})$.

\section{JAK3 Activation and the Suppression of CAMP-Dependent CFTR Expression Contribute to Acute Lung Injury Following H5N1 HA Challenge}

Acute lung injury with severe interstitial edema and infiltration of inflammatory cells was observed (using pathologic examination) in wild-type mice $12 \mathrm{~h}$ following intratracheal challenge with HA (1 mg/kg) (Figures 3B, I). In contrast, wild-type mice pretreated with forskolin or JAK3 inhibitor VI and $\mathrm{JAK}^{+/-}$mice were protected from this pulmonary inflammatory response (Figures 3C-E, I). However, pretreatment of $\mathrm{JAK}^{+/+}$mice with the CFTR inhibitor glibenclamide significantly accelerated the pulmonary inflammation triggered by a low dose of H5N1 HA $(0.5 \mathrm{mg} / \mathrm{kg})$ (Figures 3F-H, I). Corresponding to the pulmonary inflammation in the mice following intratracheal challenge with HA ( $1 \mathrm{mg} / \mathrm{kg})$, the CFTR immunofluorescence signal in the airway epithelium was completely blocked (Figure 3K) compared with control mice treated with saline (Figure 3J). Furthermore, Western blot analysis showed a significant reduction of CFTR level in the lung of HA-challenged wild-type mice (Figure 3L).

\section{Inhibition of CAMP-Dependent CFTR Is Mediated by JAK3 Activation and Enhances NF-кB Signaling}

Immunofluorescent staining and confocal microscopy examination revealed that CFTR was expressed and primarily localized to the plasma membrane in the calu-3 cells under basal conditions. In addition, Ser276-phosphorylated NF-кBp65, which indicates NF-кB activation (20), was dispersed throughout the cytoplasm (Figure 4A). After the exposure of these cells to $80 \mu \mathrm{g} \mathrm{HA} / \mathrm{mL}$ 
A

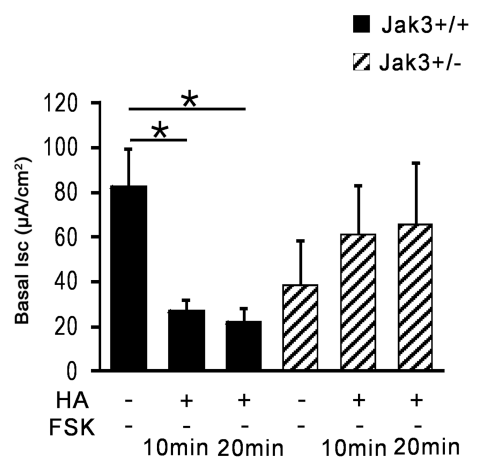

B

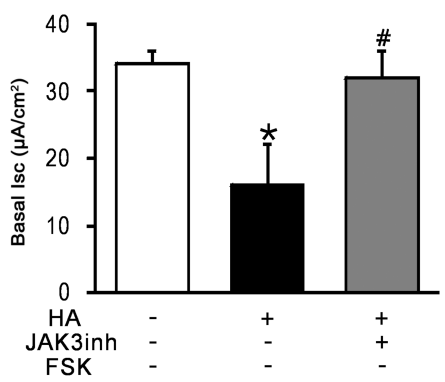

Jak3+1+

C. Jak3+l-
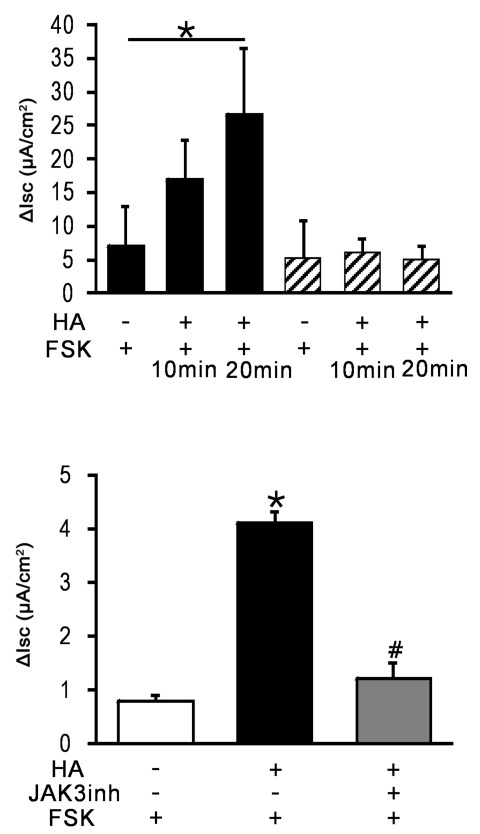

Figure 1. JAK3 activation by H5N1 HA results in the inhibition of CAMP-dependent CFTR $\mathrm{Cl}^{-}$ channels. Tracheal tissues isolated from wild-type and JAK3 gene-deficient heterozygous $\left(\mathrm{JAK3}^{+/}\right)$mice were pretreated with exposure of apical membranes to $\mathrm{H} 5 \mathrm{~N} 1 \mathrm{HA}(40 \mu \mathrm{g} / \mathrm{mL})$ for $10 \mathrm{~min}$ or $20 \mathrm{~min}$ and then mounted into a perfused micro-Ussing chamber to perform short-circuit current assay. (A) Basal short-circuit current (ISC) and changes in the shortcircuit current $(\Delta \mathrm{ISC})$ in response to forskolin (FSK, $10 \mu \mathrm{mol} / \mathrm{L})$ addition $\left(\mathrm{n}=5\right.$ per group, ${ }^{*} \mathrm{p}$ 0.05 versus $\mathrm{JAK3}^{+/+}$mice with saline). (B) Isc and $\Delta \mathrm{lsc}$ measurement in wild-type mice treated with JAK3 inhibitor VI (JAK3inh, $0.15 \mathrm{mg} / \mathrm{kg}$ ) or saline prior to exposure to HA ( $\mathrm{n}=5$ per group, ${ }^{*} p<0.05$ versus mice treated with saline; ${ }^{\#} p<0.05$ versus mice treated with $\mathrm{HA}$ ).

for $12 \mathrm{~h}$, the global CFTR immunofluorescence signal was sharply reduced (Figures 4B, G), and Ser276-phosphorylated NF-кBp65 was translocated from the cytoplasm to the nucleus (see Figure 4B). Pretreatment with either forskolin or JAK3 inhibitor VI for 30 min prior to HA $(80 \mu \mathrm{g} / \mathrm{mL})$ challenge ameliorated the reduction of the CFTR immunofluorescence signal (Figures 4C, $D, G)$, concomitant with the absence of Ser276-phosphorylated NF-кBp65 nuclear accumulation (see Figures 4C, D). However, cells stimulated with a low dose of HA $(20 \mu \mathrm{g} / \mathrm{mL})$ displayed only a slight reduction in CFTR expression (Figures 4E, G) and exhibited no visible alteration in the nuclear translocation of Ser276-phosphorylated NF-кBp65 (see
Figure 4E). However, a remarkable downregulation of CFTR expression (Figures 4F, G) concomitant with Ser276phosphorylated NF- $\mathrm{BP} 65$ activation was observed in HA (20 $\mu \mathrm{g} / \mathrm{mL})$ challenged cells pretreated with the CFTR inhibitor glibenclamide (see Figure $4 \mathrm{~F}$ ).

A bead array-based analysis was performed to examine the cytokine and chemokine levels in HA-treated 16HBE cells in the absence or presence of the JAK3 inhibitor VI. The results provide additional evidence that H5N1 HA induces the secretion of cytokines and chemokines, including ICAM-1, IL-18, IP-10, MCP-1, MIP-3 $\alpha$, MMP-7, which are related to the activation of JAK3 (Figure 5).
A

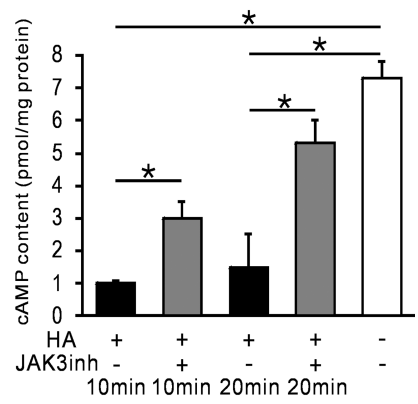

B

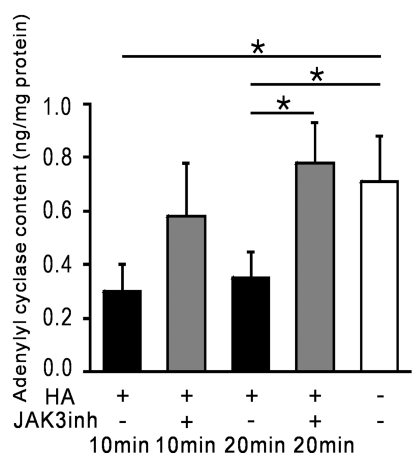

Figure 2. JAK3 activation by H5N1 HA inhibits AC activity and reduces the CAMP level. Cultured bronchial epithelial cells (16HBE) were pretreated with JAK3 inhibitor VI (JAK3inh, $760 \mathrm{nmol} / \mathrm{L}$ ) or saline for $30 \mathrm{~min}$ prior to $\mathrm{HA}(40 \mu \mathrm{g} / \mathrm{mL})$ treatment. Then, 10 min or 20 min after the HA challenge, (A) the intracellular cAMP levels and $(B)$ the adenylyl cyclase activity in these cells were evaluated via ELISA. ${ }^{*} p<$ 0.05 versus control cells treated with saline or cells treated with HA alone.

\section{H5N1 HA Is Detected by TLR4 Expressed on Airway Epithelial Cells, which Facilitates JAK3 Activation}

The cell-surface proteins Toll-like receptor (TLR)2 and TLR4, which belong to the family of pattern recognition receptors (PRRs), may interact with viral glycoprotein products (21). Therefore, we further examined the mechanism by which H5N1 HA, a pathogen-associated molecular pattern (PAMP), is sensed by TLR4 on the airway epithelial cell surface, leading to the activation of JAK3. 


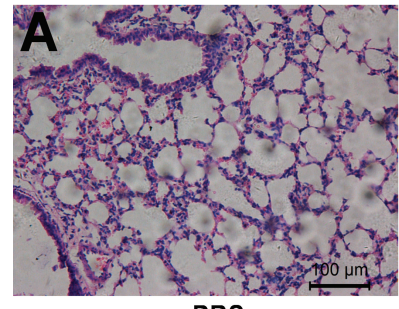

PBS

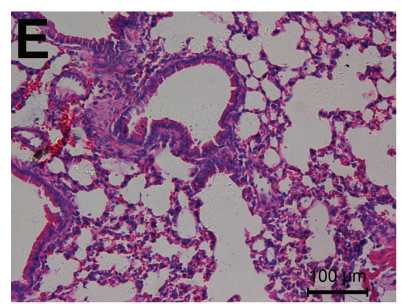

$\mathrm{HA}(\mathrm{h})+\mathrm{JAK} 3+I-$

I

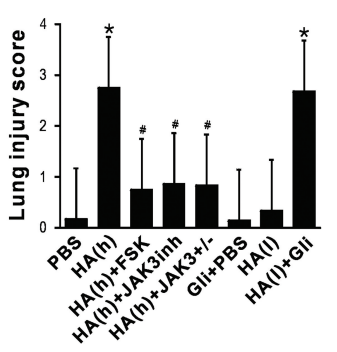

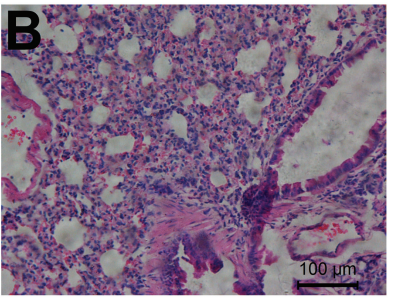

HA(h)

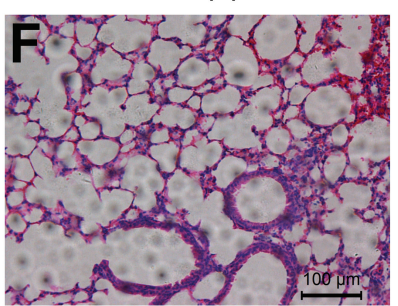

Gli+PBS

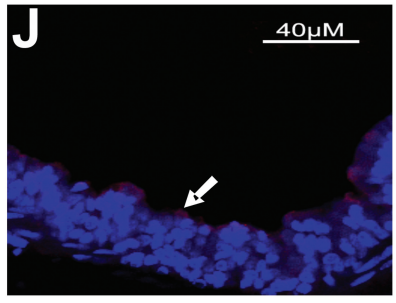

PBS

CFTR

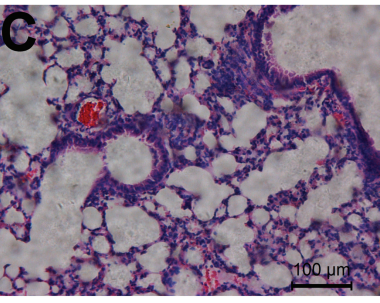

$H A(h)+F S K$

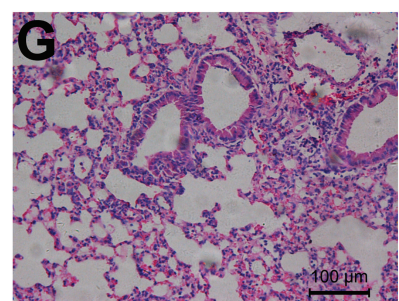

HA(I)

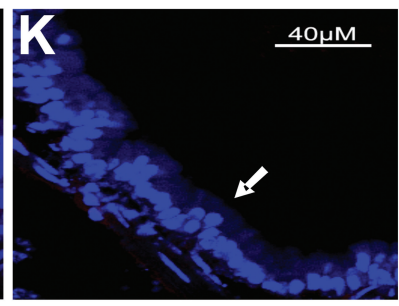

HA(h)

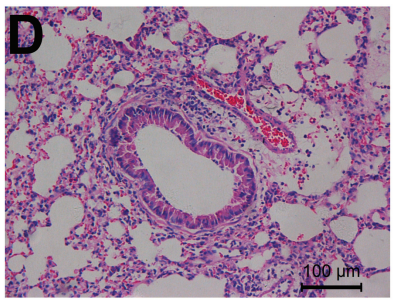

HA(h)+JAK3inh

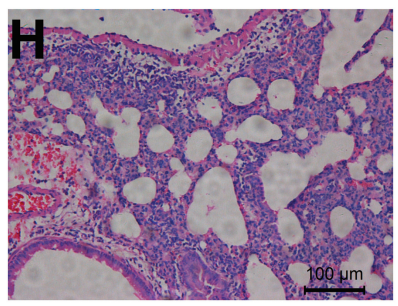

$H A(I)+G I i$



Figure 3. Blockade of CAMP-dependent CFTR channels via JAK3 activation exacerbates acute lung injury in response to H5N1 HA challenge. Histopathologic examination via hematoxylin and eosin (H\&E) staining was performed on the lung sections of JAK3 ${ }^{+/-}$and $\mathrm{JAK}^{+/+}$ mice. (A) $\mathrm{JAK3}^{+/+}$mice treated with saline; (B) with HA alone at $1 \mathrm{mg} / \mathrm{kg}$ or (G) $0.5 \mathrm{mg} / \mathrm{kg}$; (C) HA (1 mg/kg) with either forskolin (FSK, $10 \mathrm{mg} / \mathrm{kg}$ ) or (D) JAK3 inhibitor VI (JAK3inh, $0.15 \mathrm{mg} / \mathrm{kg}$ ); (F) glibenclamide pretreatment (Gli, $10 \mathrm{mg} / \mathrm{kg}$ ) alone or (H) HA $(0.5 \mathrm{mg} / \mathrm{kg}$ ) with glibenclamide pretreatment. (E) JAK3 ${ }^{+-}$mice treated with HA (1 mg/kg). Scale bars = $100 \mu \mathrm{m}$. h: high (1 mg/kg); L: low (0.5 mg/kg). (I) The lung injury score is assessed. The data are expressed as the mean \pm SEM, with $n=5$ per group. ${ }^{*} p<0.05$, versus saline control; $\# p<0.05$ versus the HA(h)-challenged $\mathrm{JAK}^{+/+}$group. $(\mathrm{J})$ Immunofluorescent staining was performed to examine CFTR expression in $\mathrm{JAK3}^{+/+}$mice treated with saline or (K) HA (1 mg/kg) for $4 \mathrm{~h}$. Scale bars $=40 \mu \mathrm{m}$. The arrows indicate positively stained cells. (L) Western blot analysis was performed to determine the level of CFTR expression. ${ }^{*} P<0.05$, versus saline control, c: control.

We verified that the exposure of the apical surface of tracheal tissues isolated from wild-type mice to HA $(40 \mu \mathrm{g} / \mathrm{mL})$ in the presence of a TLR4 inhibitor did not induce a detectable decrease in the Isc in contrast to HA exposure in the absence of the TLR4 inhibitor. The response of the tracheal tissue to forskolin following pretreatment with HA and the TLR4 inhibitor displayed a reduced $\Delta \mathrm{Isc}$ compared with tissue pretreated with HA alone (Figure 6A).

Immunofluorescence and Western blot analyses revealed that the HAstimulated activation of JAK3 in bronchial epithelial cells was blocked by the addition of either a TLR4 or JAK3 inhibitor (Figures 6B, C). A 1.5-fold and 1.8-fold increase in TLR4 gene transcription was observed at $12 \mathrm{~h}$ and $24 \mathrm{~h}$ after HA exposure, respectively, and this increase was accompanied by a significant decrease in CFTR gene expression at $12 \mathrm{~h}$ (Figure 6D).

\section{H5N1 HA Interaction with TLR4 Activates the Heterotrimeric Gai/o Protein in Airway Epithelial Cells via JAK3 and Decreases CAMP Production}

Activated heterotrimeric Gai/o proteins inhibit the function of $\mathrm{AC}$ and reduce the intracellular cAMP levels (22); therefore, we determined whether
Gai/o proteins are activated by JAK3 following H5N1 HA stimulation in $16 \mathrm{HBE}$ cells. To confirm that the decrease in the cellular cAMP levels after H5N1 stimulation was due to the increased activation of Gai/o proteins, these human airway epithelial cells were treated with a Gai/o protein inhibitor (pertussis toxin, PTx), a JAK3 inhibitor (JAK3 inhibitor VI) or a TLR4 inhibitor prior to stimulation with $\mathrm{H} 5 \mathrm{~N} 1$ HA. Treatment with PTx or JAK3 inhibitor VI completely reversed the decrease in forskolin-induced cAMP production following challenge with $\mathrm{H} 5 \mathrm{~N} 1$ $\mathrm{HA}$. The reduction in cAMP production was not observed in TLR4 inhibitor-pre- 

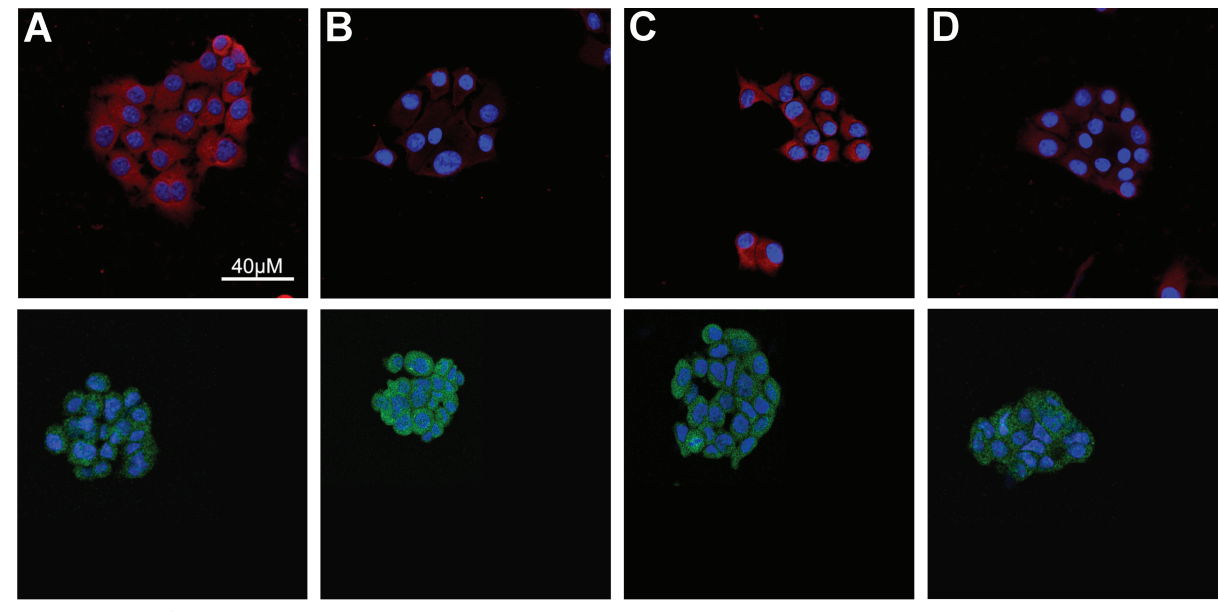

$H A(h)$
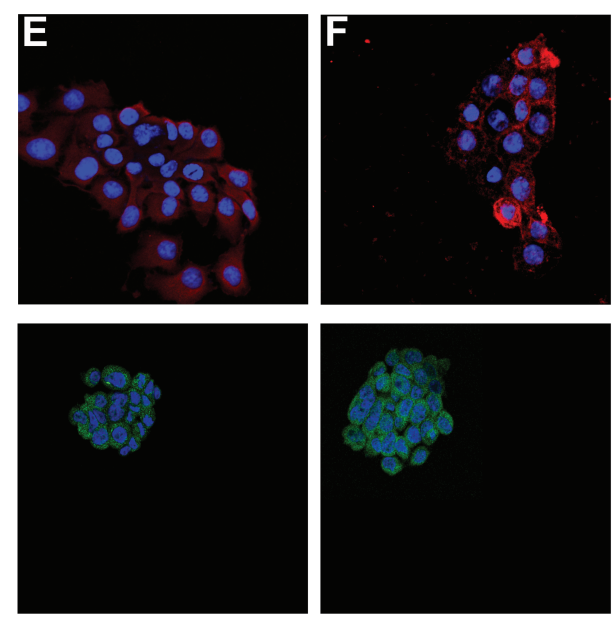

HA(I)

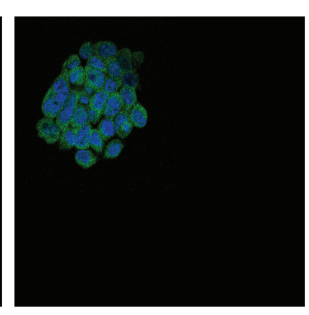

$H A(I)+G l i$

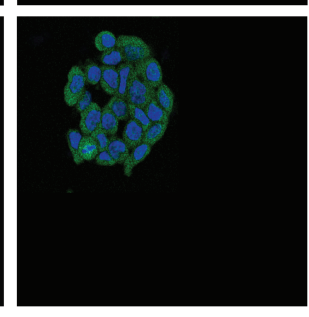

HA(h)+FSK

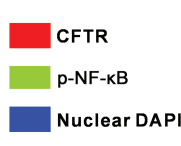

G

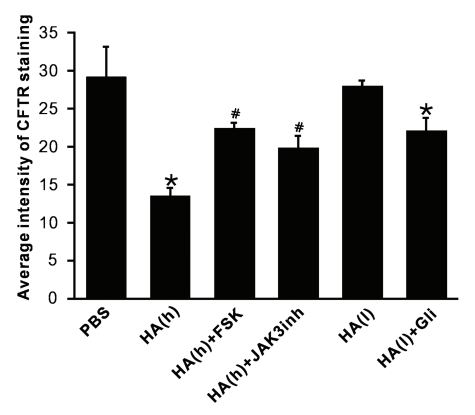

Figure 4. Inhibition of CAMP-dependent CFTR channels via JAK3 activation enhances phosphorylated NF-KBp65 nuclear translocation in bronchial cells after exposure to H5N1 HA. Immunofluorescent staining of cultured calu-3 cells was performed following HA addition at two doses for $12 \mathrm{~h}$ in the absence or presence of pretreatment with JAK3 inhibitor VI (JAK3inh, $760 \mathrm{nmol} / \mathrm{L}$ ), forskolin (FSK, $10 \mu \mathrm{mol} / \mathrm{L}$ ) or glibenclamide (Gli, $500 \mu \mathrm{mol} / \mathrm{L}$ ). (A) The

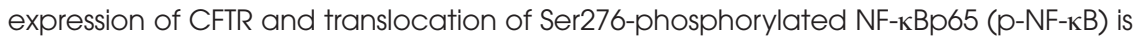
shown in calu-3 cells treated with saline; (B) HA alone at $80 \mu \mathrm{g} / \mathrm{mL}$ or (E) $20 \mu \mathrm{g} / \mathrm{mL}$; (C) HA $(80 \mu \mathrm{g} / \mathrm{mL})$ following pretreatment with either forskolin or (D) JAK3 inhibitor Vl; or (F) glibenclamide pretreatment followed by treatment with HA at $20 \mu \mathrm{g} / \mathrm{mL}$. Scale bars $=40 \mu \mathrm{m} \mathrm{h}$ : high $(80 \mu \mathrm{g} / \mathrm{mL})$, L: low $(20 \mu \mathrm{g} / \mathrm{mL})$. (G) Average fluorescence intensity of CFTR staining is represented. The data are expressed as the mean \pm SEM, with $n=3$ to 5 per group. ${ }^{*} p<$ 0.05 versus saline control; ${ }^{\#} p<0.05$ versus the cells with HA (h) treatment alone.

treated cells. In addition, the simultaneous addition of IL-2, a JAK3 agonist, with H5N1 HA ameliorated the effects on CAMP production caused by pretreatment with JAK3 inhibitor VI. These results demonstrate that Gai/o proteins are activated by JAK3 following H5N1 HA stimulation in airway epithelial cells, contributing to the inhibition of the activity of AC, thereby reducing cellular cAMP production (Figure 7).

\section{DISCUSSION}

Our findings provide novel insight into the pathogenesis of H5N1-induced acute lung injury via the inhibition of airway epithelium cAMP-dependent CFTR $\mathrm{Cl}^{-}$channels, which is associated with host tolerance to infection. We report that H5N1 HA-triggered JAK3 activation inhibits $A C$, the enzyme exclusively responsible for cAMP production (23). This inhibition induces a defect in cAMP production, thereby blocking cAMP-dependent CFTR channels.

A reduction in barrier function due to the impairment of CFTR-mediated trafficking caused by H5N1 HA exposure may facilitate the entry of viral or bacterial toxins into the submucosa, increasing the susceptibility of the host to pathogen infection (24-27).

Previous studies reported that an increase in cAMP due to the administration of forskolin or dibutyryl-cAMP reduced JAK3 expression, and this reduction resulted in impaired IL-2dependent signal transduction and inhibition of T-cell activation (28). Consistently, targeting to JAK3 by the administration of JAK3 inhibitor VI alleviated acute lung injury (17). Current evidence provides strong support for the hypothesis that JAK3 activation via H5N1 HA attachment, which is associated with reduced CAMP production and suppression of cAMP-dependent CFTR $\mathrm{Cl}^{-}$channels in the airway epithelium, leads to an enhanced inflammatory reaction.

Recent observational data reported in an investigation of primary lower airway epithelial cells (AECs) in children with CF suggest a possible nexus between human rhinovirus (HRV) infection and AEC-initiated inflammatory cell recruitment and activation, thus leading to early airway inflammation (29). In the present study, we demonstrated an exaggerated inflammatory response in the lung tissue of HA-challenged wild-type mice following pretreatment with a CFTR channel inhibitor. These results are consistent with previous studies suggesting slower viral clearance, increased severity of infection and a prolonged inflammatory response to respiratory viral infection in CF $(30,31)$.

Accumulating evidence has indicated that alterations in intracellular $\mathrm{Cl}^{-}$concentrations play an important role in a 
A

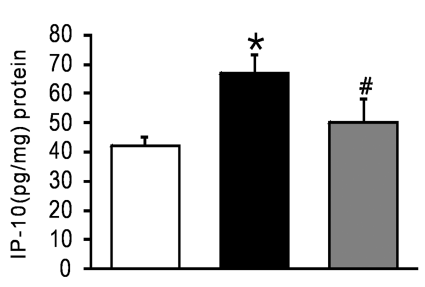

C

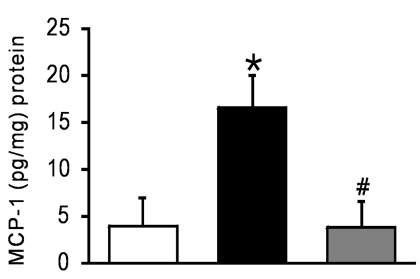

$\mathbf{E}$

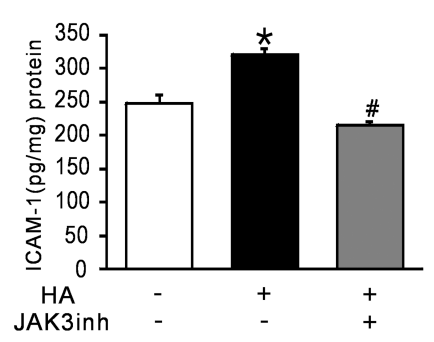

B



D

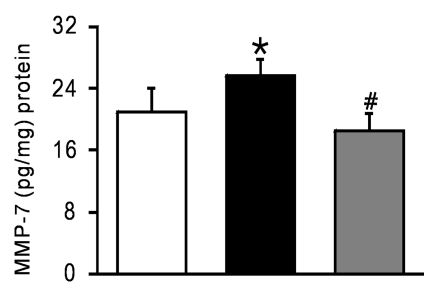

$\mathbf{F}$

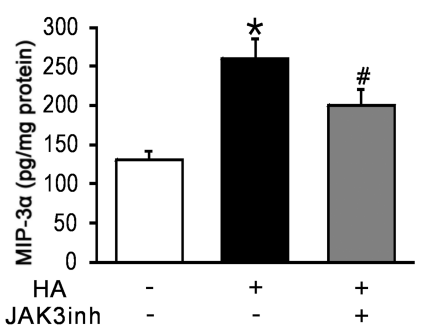

Figure 5. Inhibiting JAK3 results in a reduction in cytokine/chemokine hyperproduction. Cultured 16HBE cells were incubated with HA $(40 \mu \mathrm{g} / \mathrm{mL})$ for $12 \mathrm{~h}$ with or without JAK3 inhibitor VI (JAK3inh, $760 \mathrm{nmol} / \mathrm{L})$ pretreatment, and the culture supernatants were subjected to a LiquidChip assay. ${ }^{*} p<0.05$ versus saline control cells; ${ }^{\#} p<0.05$ versus cells incubated with HA alone.

variety of physiological and pathological processes (32-34). Yang et al. recently reported that a decrease in the intracellular $\mathrm{Cl}^{-}$concentration promotes endothelial cell inflammation by activating the NF-кB pathway (35). Consistent with this result, our findings show that the attenuation of the CFTR $\mathrm{Cl}^{-}$channel by glibenclamide exacerbated $\mathrm{H} 5 \mathrm{~N} 1$ HA-induced acute lung injury, which was associated with enhanced NF- $\mathrm{B}$ translocation. These data indicate that a reduction in CFTR channel-dependent $\mathrm{Cl}^{-}$transport underlies, at least in part, the HA-induced superinflammation. By contrast, in the presence of forskolin, which is commonly used to open CFTR channels by increasing cAMP levels, or a JAK3 inhibitor, bronchial epithelial calu-3 cells display reduced activation of NF-кB p65 induced by HA challenge. Therefore, the CFTR $\mathrm{Cl}^{-}$channel may play an essential role in the regulation of the innate immune inflammatory response by dynamically modulating $\mathrm{Cl}^{-}$movement across the plasma membrane.

NF- $\kappa$ B plays a crucial role in inducing the expression of a plethora of inflammatory and immune mediators; thus, $\mathrm{NF}-\kappa \mathrm{B}$ is one of the master regulators of the immune response and a key target for antiinflammatory drug design. A number of fundamental molecular mechanisms that contribute to the overall inhibitory action of cAMP on NF- $\mathrm{BB}$ function are well established (36). In the presence of forskolin and a JAK3 in- hibitor, both wild-type mice and bronchial epithelial cells counteract NF- $\kappa \mathrm{B}$ activation and inflammatory damage in response to HA challenge, indicating that interactions between cAMP and the NF- $\mathrm{B}$ signaling cascade modulate the outcome of inflammationassociated NF- $\kappa \mathrm{B}$ activation, which are modulated by JAK3 expression.

The activity of AC is modulated by G-protein subunits. Specifically, Gas stimulates AC, inducing it to catalyze the formation of cAMP from ATP, whereas the Gai protein exerts an inhibitory effect on AC. Recent studies have shown that TLR ligands activate Gai proteins in endothelial cells $(22,37)$. The authors speculated that TLR2, 3 and 4 also interact directly with Gai via their intracellular domains due to a consensus motif for Gai/o binding. Consistently, our results indicate that cytoplasmic JAK3 activation evokes the interaction between TLR4 and Gai via their intracellular binding domains, leading to the inhibition of AC and reducing cAMP production. JAK3 activation, which is after activation of TLR4 by H5N1 HA, results in impaired cAMP-dependent CFTR regulation and CFTR gene expression, contributing to the exacerbation of immune inflammation. Therefore, we suggest that airway epithelial cAMP-dependent CFTR regulation represents an important host tolerance mechanism for limiting tissue damage to prevent severe immunopathology and that cAMP levels and CFTR function are closely modulated by JAK3 activity, which exerts an inhibitory effect on AC by activating Gai G-protein subunits. Hence, cAMPmodulating therapeutic strategies potentially hold promise for treating ARDS induced by avian influenza virus infection; however, further investigation is required.

\section{CONCLUSION}

In conclusion, we suggest that the inhibition of cAMP-dependent CFTR channels by TLR4-stimulated JAK3 activation, which blocks AC-mediated cAMP pro- 
A
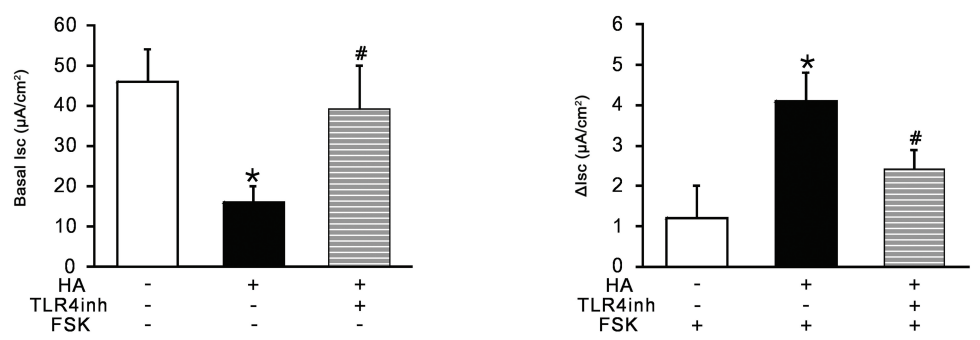

B

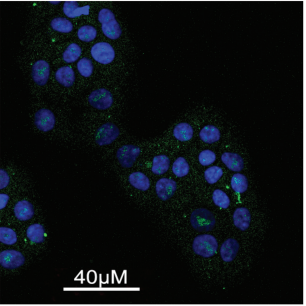

PBS

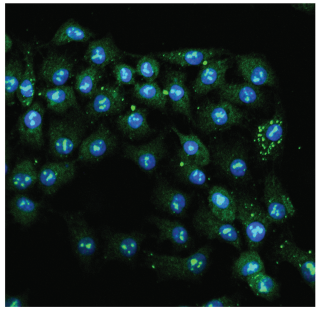

HA

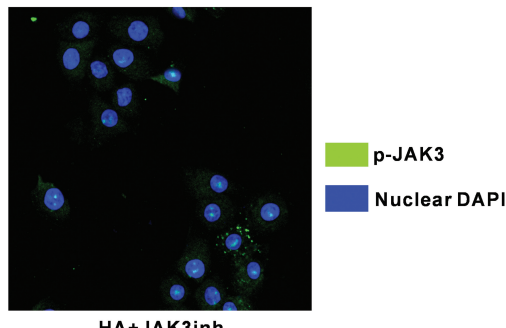

C
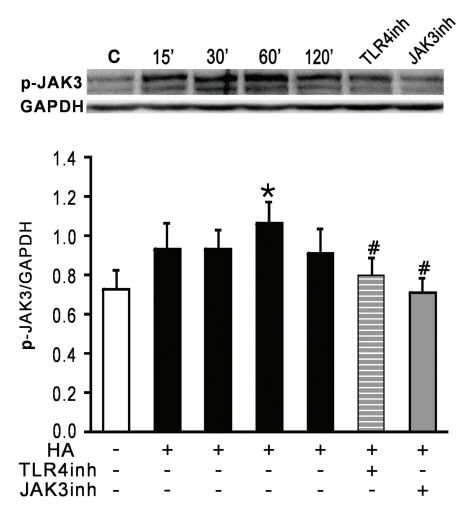

D

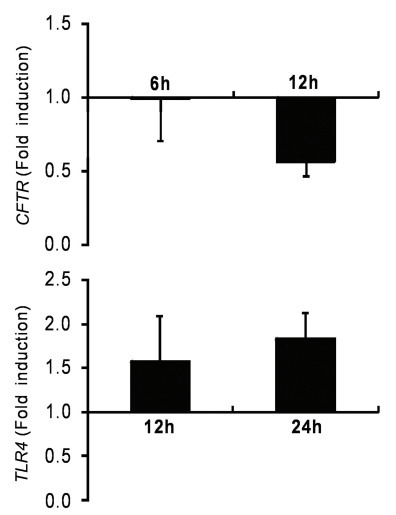

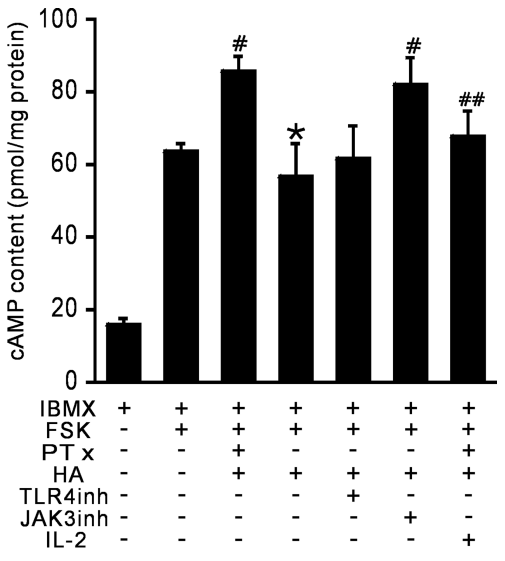

Figure 7. HA-induced JAK3 activation stimulates Gai-mediated inhibition of AC, resulting in a decrease in the intracellular cAMP level 16HBE cells were pretreated with IBMX (1 mmol/L )for $15 \mathrm{~min}$ and then treated with forskolin (FSK, $10 \mu \mathrm{mol} / \mathrm{L}, 30$ min) and either $\mathrm{HA}(40 \mu \mathrm{g} / \mathrm{mL}, 30 \mathrm{~min})$ alone or $\mathrm{HA}(40 \mu \mathrm{g} / \mathrm{mL}, 30 \mathrm{~min})$ in combination with IL-2 (100 UI/mL, 30 min) as indicated. In some instances, cells were pretreated with pertussis toxin (PTx, $100 \mathrm{ng} / \mathrm{mL}$ ) for $16 \mathrm{~h}$ or a TLR4 inhibitor (TLR4inh, $5 \mu \mathrm{mol} / \mathrm{L}$ ) or JAK3 inhibitor VI (JAK3inh, $760 \mathrm{nmol} / \mathrm{L}$ ) for $30 \mathrm{~min}$. The intracellular cAMP levels were examined in the cell lysates. " $p<0.05$ versus forskolintreated cells; ${ }^{\#} p<0.05$ versus HA-treated cells; and ${ }^{\# \#} p<0.05$ versus PTx-treated cells.

for help with experiments. This work was supported by grants from the National Key Basic Research Program of China (973 Program; 2009CB522104), the National Natural Science Foundation of China (30900576), the China Postdoctoral Science Foundation (20100480734), the Yangcheng Scholars Research Program of Guangzhou Municipal Universities (10A024G) and the Program for Tackling Key Problems in Science and Technology of the Local Government in Guangzhou (2014), China.

VI) may represent an effective therapeutic strategy for the management of influenza virus-induced severe pneumonia. acerbation of innate immune inflammatory responses. Increasing the intracellular cAMP levels via the administration of cAMP-elevating agents and overcoming the attenuation of CAMP production by selective JAK3 inhibition (JAK3 inhibitor

\section{ACKNOWLEDGMENTS}

The authors would like to thank Wenliang Zhou (School of Life Science, Sun Yat-sen University, Guangzhou, China)

\section{DISCLOSURES}

The authors declare that they have no competing interests as defined by Molecular Medicine or other interests that might be perceived to influence the results and discussion reported in this paper. 


\section{REFERENCES}

1. Ramos I, Fernandez-Sesma A. (2012) Innate immunity to H5N1 influenza viruses in humans. Viruses. 4:3363-88.

2. Peiris JSM, Cheung CY, Leung CYH, Nicholls JM. (2009) Innate immune responses to influenza A H5N1: friend or foe? Trends Immunol. 30:574-84.

3. Schmid-Hempel P. (2011) Evolutionary Parasitology: The Integrated Study of Infections, Immunology, Ecology, and Genetics. Oxford: Oxford University Press. 516 pp.

4. Casadevall A, Pirofski L-a. (1999) Host-pathogen interactions: redefining the basic concepts of virulence and pathogenicity. Infect. Immun. 67:3703-13.

5. Medzhitov R, Schneider DS, Soares MP. (2012) Disease tolerance as a defense strategy. Science. 335:936-41.

6. Majno G, Joris I. (2004) Cells, Tissues, and Disease: Principles of General Pathology. 2nd edition. New York: Oxford University Press. 1005 pp.

7. Nash AA, Dalziel RG, Fitzgerald JR. (2015) Mims' Pathogenesis of Infectious Disease. Amsterdam: Academic Press. $356 \mathrm{pp}$

8. Caputo A, et al. (2008) TMEM16A, a membrane protein associated with calcium-dependent chloride channel activity. Science. 322:590-4.

9. Stutts MJ, et al. (1995) CFTR as a cAMPdependent regulator of sodium channels. Science. 269:847-50.

10. Tarran R, Button B, Boucher RC. (2006) Regulation of normal and cystic fibrosis airway surface liquid volume by phasic shear stress. Annu. Rev. Physiol. 68:543-61.

11. Venkatakrishnan A, et al. (2000) Exaggerated activation of nuclear factor-kappaB and altered IkappaB-beta processing in cystic fibrosis bronchial epithelial cells. Am. J. Respir. Cell Mol. Biol. 23:396.

12. Cohen TS, Prince A. (2012) Cystic fibrosis: a mucosal immunodeficiency syndrome. Nat. Med. 18:509-19.

13. Cohen-Cymberknoh M, Kerem E, Ferkol T, Elizur A. (2013) Airway inflammation in cystic fibrosis: molecular mechanisms and clinical implications. Thorax. 68:1157-62.

14. Rogers G, et al. (1983) Single amino acid substitutions in influenza haemagglutinin change receptor binding specificity. Nature. 304:76-8.

15. Salomon R, Webster RG. (2009) The influenza virus enigma. Cell. 136:402-10.

16. Liu W-C, Lin S-C, Yu Y-L, Chu C-L, Wu S-C. (2010) Dendritic cell activation by recombinant hemagglutinin proteins of H1N1 and H5N1 influenza A viruses. J. Virol. 84:12011-7.

17. Xu W, Chen M, Ge N, Xu J. (2012) Hemagglutinin from the $\mathrm{h} 5 \mathrm{n} 1$ virus activates Janus kinase 3 to dysregulate innate immunity. PloS One. 7:e31721.

18. Committee for the Update of the Guide for the Care and Use of Laboratory Animals, Institute for Laboratory Animal Research, Division on Earth and Life Studies, National Research Council of the National Academies. (2011) Guide for the
Care and Use of Laboratory Animals. 8th edition. Washington (DC): National Academies Press.

19. Goddard CA, Evans MJ, Colledge WH. (2000) Genistein activates CFTR-mediated $\mathrm{Cl}$ - secretion in the murine trachea and colon. Am. J. Physiol. Cell Physiol. 279:C383-92.

20. Vermeulen L, De Wilde G, Notebaert S, Haegeman G. (2002) Regulation of the transcriptional activity of the nuclear factor-kappaB p65 subunit. Biochem. Pharmacol. 64:963-70.

21. Bowie AG, Haga IR. (2005) The role of Toll-like receptors in the host response to viruses. Mol. Immunol. 42:859-67.

22. Dauphinee SM, Voelcker V, Tebaykina Z, Wong F, Karsan A. (2011) Heterotrimeric Gi/Go proteins modulate endothelial TLR signaling independent of the MyD88-dependent pathway. Am. J. Physiol. Heart Circ. Physiol. 301:H2246-53.

23. Billington CK, Hall IP. (2012) Novel cAMP signalling paradigms: therapeutic implications for airway disease. Br. J. Pharmacol. 166:401-10.

24. Morimoto K, et al. (1998) Influence of absorption enhancers (bile salts) and the preservative (benzalkonium chloride) on mucociliary function and permeation barrier function in rabbit tracheas. Eur. J. Pharm. Sci. 6:225-30.

25. Boucher R. (2007) Evidence for airway surface dehydration as the initiating event in CF airway disease. J. Int. Med. 261:5-16.

26. Humlicek AL, et al. (2007) Paracellular permeability restricts airway epithelial responses to selectively allow activation by mediators at the basolateral surface. J. Immunol 178:6395-403.

27. LeSimple P, Liao J, Robert R, Gruenert DC, Hanrahan JW. (2010) Cystic fibrosis transmembrane conductance regulator trafficking modulates the barrier function of airway epithelial cell monolayers. J. Physiol. 588:1195-209.

28. Kolenko V, et al. (1999) Downregulation of JAK3 protein levels in $\mathrm{T}$ lymphocytes by prostaglandin E2 and other cyclic adenosine monophosphateelevating agents: impact on interleukin-2 receptor signaling pathway. Blood. 93:2308-18.

29. Sutanto EN, et al. (2011) Innate inflammatory responses of pediatric cystic fibrosis airway epithelial cells: effects of nonviral and viral stimulation. Am. J. Respir. Cell Mol. Biol. 44:761-7.

30. Wang EE, Prober CG, Manson B, Corey M, Levison H. (1984) Association of respiratory viral infections with pulmonary deterioration in patients with cystic fibrosis. N. Eng. J. Med. 311:1653-8.

31. Colasurdo GN, et al. (2006) Respiratory syncytial virus infection in a murine model of cystic fibrosis. J. Med. Virol. 78:651-8.

32. Wang XQ, et al. (2006) CLC-3 channels modulate excitatory synaptic transmission in hippocampal neurons. Neuron. 52:321-33.

33. Hong L, et al. (2011) Alteration of volumeregulated chloride channel during macrophagederived foam cell formation in atherosclerosis. Atherosclerosis. 216:59-66.

34. Heimlich G, Cidlowski JA. (2006) Selective role of intracellular chloride in the regulation of the intrinsic but not extrinsic pathway of apoptosis in Jurkat T-cells. J. Biol. Chem. 281:2232-41.

35. Yang H, et al. (2012) Decrease of intracellular chloride concentration promotes endothelial cell inflammation by activating nuclear factor- $\kappa \mathrm{B}$ pathway. Hypertension. 60:1287-93.

36. Gerlo S, et al. (2011) Cyclic AMP: a selective modulator of NF-кB action. Cell. Mol. Life Sci. 68:3823-41.

37. Hildebrand D, Sahr A, Wölfle SJ, Heeg K, Kubatzky KF. (2012) Regulation of Toll-like receptor 4-mediated immune responses through Pasteurella multocida toxin-induced $\mathrm{G}$ protein signalling. Cell. Commun. Signal 10:22.

Cite this article as: Cao K, et al. (2015) H5N1 virus hemagglutinin inhibition of cAMP-dependent CFTR via TLR4-mediated Janus tyrosine kinase 3 activation exacerbates lung inflammation. Mol. Med. 21:134-42. 